\title{
Pressure-induced two-step spin transition with structural symmetry breaking: $X$-ray diffraction, magnetic, and Raman studies
}

Shepherd, H.J.; Bonnet, S.A.; Guionneau, P.; Bedoui, S.; Garbarino, G.; Nicolazzi, W.; ... ; Molnar, G.

\section{Citation}

Shepherd, H. J., Bonnet, S. A., Guionneau, P., Bedoui, S., Garbarino, G., Nicolazzi, W., ... Molnar, G. (2011). Pressure-induced two-step spin transition with structural symmetry breaking: X-ray diffraction, magnetic, and Raman studies. Physical Review B, 84(14), 144107. doi:10.1103/PhysRevB.84.144107

Version: $\quad$ Not Applicable (or Unknown)

License: $\quad$ Leiden University Non-exclusive license

Downloaded from: https://hdl.handle.net/1887/61366

Note: To cite this publication please use the final published version (if applicable). 


\title{
Pressure-induced two-step spin transition with structural symmetry breaking: X-ray diffraction, magnetic, and Raman studies
}

\author{
Helena J. Shepherd, ${ }^{1,2}$ Sylvestre Bonnet ${ }^{3}$ Philippe Guionneau, ${ }^{2}$ Salma Bedoui, ${ }^{1}$ Gaston Garbarino, ${ }^{4}$ William Nicolazzi, ${ }^{1}$ \\ Azzedine Bousseksou, ${ }^{1}$ and Gábor Molnár ${ }^{1, *}$ \\ ${ }^{1}$ Laboratoire de Chimie de Coordination, CNRS UPR-8241, and Université de Toulouse, UPS, INP, Toulouse, France \\ ${ }^{2}$ CNRS, Université de Bordeaux, ICMCB, 87 avenue du Dr A. Schweitzer, F-33608 Pessac, France \\ ${ }^{3}$ Leiden Institute of Chemistry, Leiden University, P.O. Box 9502, 2300 RA Leiden, The Netherlands \\ ${ }^{4}$ European Synchrotron Radiation Facility (ESRF), BP 220, 6 Rue Jules Horowitz, 38043 Grenoble Cedex, France
}

(Received 23 June 2011; published 10 October 2011)

\begin{abstract}
We have used single-crystal x-ray diffraction, Raman spectroscopy, and magnetometry to investigate the effect of hydrostatic pressures up to $16 \mathrm{kbar}$ on the molecular spin crossover complex [Fe (II (bapbpy)(NCS) $)_{2}$. A stepped first-order transition from the high-spin (HS) to low-spin (LS) phase was observed upon compression of single-crystal samples. The intermediate phase (IP) is stable between 4 and $11 \mathrm{kbar}$ at room temperature. This phase is characterized by supercell reflections and tripling of the $c$-axis of the unit cell $(\mathrm{C} 2 / \mathrm{c})$ due to the formation of a periodic [HS-LS-LS] structural motif, as seen in the thermal stepped transition. The pressure-temperature phase diagram reveals an anomalous increase of the thermal hysteresis widths with increasing pressure and the stabilization of the IP across the investigated $P-T$ space.
\end{abstract}

DOI: 10.1103/PhysRevB.84.144107

PACS number(s): 75.30.Wx, 61.50.Ks, 62.50.-p, 31.70.Ks

\section{INTRODUCTION}

Intriguing two-step thermal high-spin $\rightarrow$ low-spin transitions in which the temperature dependence of the order parameter, $\mathrm{n}_{\mathrm{HS}}(\mathrm{T})$ - the high-spin fraction-displays a plateau at values of temperature for which $0<\mathrm{n}_{\mathrm{HS}}<1$ have been reported for a number of mononuclear ${ }^{1}$ and multinuclear spin crossover (SCO) molecular complexes. ${ }^{2-8}$ Mono- or multinuclear systems that have more than one crystallographically distinct SCO site prior to a stepped transition may be merely interpreted in terms of the individual sites having different chemical environments and/or intermolecular interactions and hence different ligand field and/or interaction parameters. That such metal sites may undergo SCO at somewhat different temperatures is certainly no surprise.

However, complexes in which crystallographically equivalent metal centers undergo stepped SCO have also been observed, and several theoretical models have been applied to explain such a phenomenon. For example, the complex $\left[\mathrm{Fe}\left(2\right.\right.$-pic) $\left.{ }_{3}\right] \mathrm{Cl}_{2} \cdot \mathrm{EtOH}$ (where 2-pic $=2$-picolylamine) is a well-studied mononuclear SCO material in which the stepped spin transition shows a concomitant re-entrant phase transition with an ordered HS-LS intermediate phase (IP). ${ }^{9}$ Subsequently several other examples of thermally induced stepped SCO accompanied by spontaneous crystallographic symmetry breaking have been reported, ${ }^{10-20}$ revealing what was once thought to be a singular case of unusual SCO behavior might in fact be a rule. It is worth mentioning that a distinction should be made between magnetic symmetry breaking and crystallographic symmetry breaking accompanying the spin transition. The term "crystallographic symmetry breaking" is used here to refer to a change in symmetry of the entire system leading to long-range order of HS and LS sites in the intermediate phase. By contrast, magnetic symmetry breaking may refer only to a breaking of symmetry at the local scale, for example, in binuclear systems and makes no predictions as to the presence or absence of long-range order in the material. $^{21}$
The application of an external pressure may allow for the fine-tuning of energy gaps $(P \Delta V)$ as well as for decoupling the crystallographic phase and spin-state transitions. ${ }^{22}$ For example, in the $\left[\mathrm{Fe}(2-\mathrm{pic})_{3}\right] \mathrm{Cl}_{2} \cdot \mathrm{EtOH}$ complex, the temperatureinduced spin transition does not show a step at $1.35 \mathrm{kbar}$ applied pressure. ${ }^{23}$ While thermally induced stepped spin transitions have been observed at elevated pressures, such a transition has never been observed in the isothermal regime purely as a function of pressure. A stepped transition with crystallographic symmetry breaking has not been observed in either regime; it is expected that increasing pressure will result in more gradual SCO behavior and thus possibly preclude such symmetry-breaking phase transitions. ${ }^{24}$ However, it is certainly possible that the lack of such an observation is merely a result of the paucity of high-pressure structural studies in this field. ${ }^{25}$ Indeed, the majority of high-pressure investigations involving SCO systems have used spectroscopic techniques or magnetic studies to characterize the effect of applied pressure on the spin transition. ${ }^{26}$ Very few studies to date have used diffraction techniques to explore the relationship between structure and properties of SCO materials under pressure, ${ }^{27-30}$ but such investigations are essential to establishing the driving forces behind the pressure-induced spin transition, as well as to thorough investigation of more complex phenomena such as stepped transitions.

We present here a combined Raman spectroscopy, magnetometry, and $\mathrm{x}$-ray diffraction study at high pressure for the investigation of a two-step SCO material, namely, $\left[\mathrm{Fe}(\right.$ bapbpy $\left.)(\mathrm{NCS})_{2}\right](\mathbf{1})$, where bapbpy $=6,6^{\prime}$-bis(amino-2pyridyl)-2,2'-bipyridine (Fig. 1). This mononuclear complex is known to undergo a two-step thermal spin transition involving an ordered [HS-LS-LS] intermediate phase. ${ }^{18,19}$ Both steps involved in the spin transition are first order, show hysteresis associated with nucleation and domain growth phenomena, ${ }^{31}$ and are accompanied by a crystallographic phase transition. In the intermediate phase one-third of the iron sites are in the HS state, and the remaining sites are LS. The structure of the molecule at 295,190 , and $110 \mathrm{~K}$ has been previously 

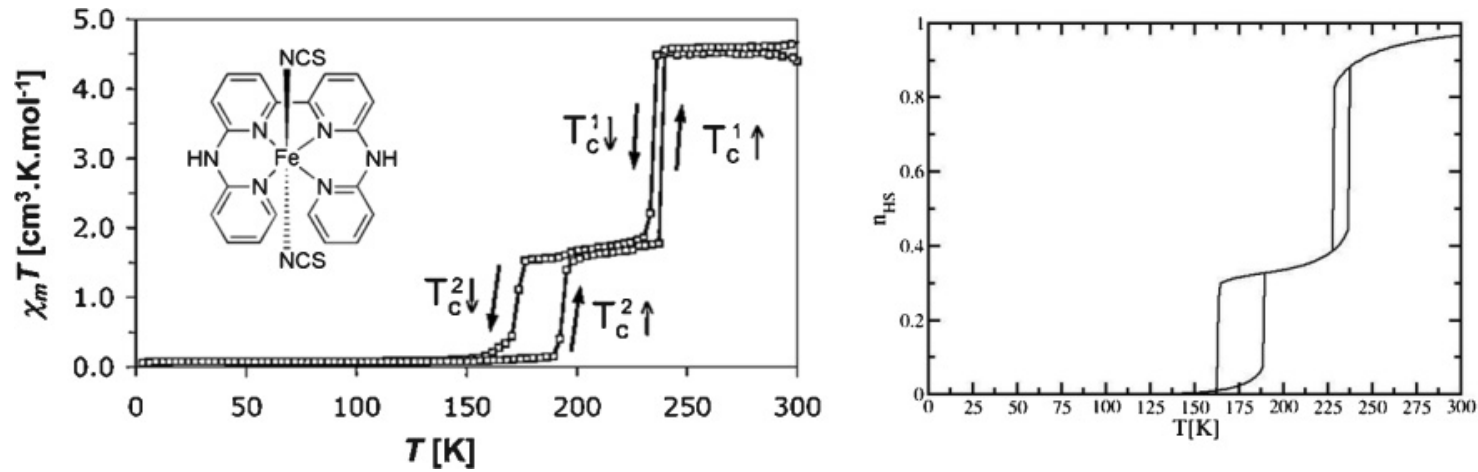

FIG. 1. Molecular structure of [Fe(bapbpy)(NCS $\left.)_{2}\right](\mathbf{1})$ and its two-step thermal spin transition curve at atmospheric pressure. Left panel: experimental data and right panel: curve simulated using the Ising-like Hamiltonian with three coupled sublattices proposed in Ref. 19.

determined by single crystal $\mathrm{x}$-ray diffraction and reported in the literature by S. Bonnet et al. ${ }^{18}$ The HS phase is in the space group $C 2 / c$; on undergoing the first spin transition, the volume of the unit cell increases by a factor of three, increasing the number of molecules in the asymmetric unit from half a HS molecule ( $Z^{\prime}=\frac{1}{2} \mathrm{HS}$ ) to half a HS molecule and one $\mathrm{LS}\left(\mathrm{Z}^{\prime}=\frac{1}{2} \mathrm{HS}, 1 \mathrm{LS}\right)$. The space group of this intermediate phase (IP) is also $C 2 / c$. During the second transition the space group changes to $C \overline{1}$ (a nonstandard, centered setting of the triclinic space group $P \overline{1}$ ) and the asymmetric unit contains one LS molecule $\left(Z^{\prime}=1 \mathrm{LS}\right)$. This final phase includes twinning involving a twofold rotation around the $b$-axis of the C-centered cell (the [110] direction in the primitive $P \overline{1}$ setting). ${ }^{33}$ Unlike in the case of $\left[\mathrm{Fe}(2-\text { pic })_{3}\right] \mathrm{Cl}_{2} \cdot \mathrm{EtOH}$, where the HS and LS states are isostructural, the $\mathrm{HS} \rightarrow \mathrm{IP} \rightarrow \mathrm{LS}$ series of crystallographic phase transitions in $\mathbf{1}$ observed on cooling is not re-entrant. Previous investigations of the effect of pressure on the SCO properties of a powder sample of this material using superconducting quantum interference device (SQUID) magnetometry revealed that while the first step of the spin transition is shifted to higher temperatures on the application of pressure, the second step is supressed. ${ }^{19}$ It should, however, be noted that the curves of the ambient pressure single crystal and powder samples are not exactly the same ${ }^{19}$ the nature (powder/single crystal) of the sample has a strong impact on the SCO properties, even at ambient pressure.

\section{EXPERIMENTAL METHODS}

A small screw-driven Diamond Anvil Cell (DAC) was used to generate pressure (at ambient temperature). It was equipped with either conical cut diamonds ${ }^{34}$ for experiments involving crystal structure refinement or Drukker (simple) cut diamonds for Raman spectroscopic studies and measurement of lattice parameters. Both sets of diamonds have 800- $\mu \mathrm{m}$ culets and are supported by tungsten carbide support seats, allowing for an X-ray aperture of approximately $80^{\circ}$ and $40^{\circ}$, respectively. In all experiments the general procedure for sample loading was the same. A steel gasket was preindented to a thickness of between 150 and $200 \mu \mathrm{m}$, and a hole of $350 \mu \mathrm{m}$ was mechanically drilled through the center of the indentation to form a sample chamber. The chamber was filled with a single crystal of $\mathbf{1}$, two small ruby chips, and Fluorinert
(FC-77) oil, which served as a pressure transmitting medium within the chamber to provide a hydrostatic environment at the sample. In all cases, pressure was incrementally increased by manually tightening each of the six screws on the DAC; it was not possible to decrease pressure in this manner. Pressure determination inside the cell was carried out using the standard ruby fluorescence method. ${ }^{35}$ Due to the resolution of the spectrometer, errors on all measured values of pressure are estimated to be \pm 0.5 kbar.

For high-pressure x-ray diffraction measurements, the DAC was mounted on a standard Huber goniometer head. Diffraction data were collected using graphite monochromated Mo- $\mathrm{K}_{\alpha}$ radiation $(\lambda=0.71073 \AA)$ on an Oxford Diffraction Gemini diffractometer equipped with an EoS detector. Determination and refinement of cell parameters and integration of diffracted intensities (where appropriate) was carried out with CrysAlisPro. ${ }^{36}$ Basic models derived from the ambient pressure phases were used as a starting point for refinement of structural parameters against high-pressure data on $F^{2}$ using SHELXL-97 ${ }^{37}$ and Olex $2 .{ }^{38}$ An absorption correction for the DAC and shadowing from the gasket was applied with Absorb 6.1. ${ }^{39}$ Anisotropic temperature factors were refined for all non-hydrogen atoms, although restraints on these were applied for $\mathrm{C}$ and $\mathrm{N}$ atoms, necessary due to low data completeness. This is reflected in the relatively high number of restraints used in the refinements. The restrictions of the steel body of the DAC mean that access to the reciprocal space is severely limited. The low $(2 / \mathrm{m})$ symmetry of this sample results in substantially incomplete data $\left(<65 \%\right.$ to $\left.50^{\circ} 2 \theta\right)$, as reflected in the comparatively poor refinement statistics. This fact, while not unusual for high-pressure single crystal data sets from small molecule (low symmetry) systems, ${ }^{40}$ means that a somewhat high number of restraints are necessary. A diffraction experiment was performed at ambient pressure with the crystal inside the cell to allow direct comparison to the previously published structure at ambient pressure and temperature.

Further combined variable pressure and temperature diffraction experiments were performed on beamline ID27 at the European Synchrotron Radiation Facility (ESRF; x-ray wavelength $=0.3738 \AA$ ), using a MAR 345 image-plate detector and a beam focused to a diameter of approx. $3 \mu \mathrm{m}$. The sample was enclosed in a gas membrane-driven $\mathrm{CuBe}$ DAC (culet diameter $600 \mu \mathrm{m}$ ) using a steel gasket; helium 
gas was used as the pressure transmitting medium. The cell was held in a helium cryostat for cooling the sample or in a heating collar for measurements above room temperature. Pressure determination was performed in situ using the ruby fluorescence technique. ${ }^{35}$ (The pressure variation was less than 1 kbar while cooling from $300 \mathrm{~K}$ to $100 \mathrm{~K}$.) Collected data at several values of pressure and temperature were indexed using CrysAlisPro. ${ }^{36}$ Analysis of cell parameters allowed phase identification by comparison to the cell parameters of the thermal HS, IP, and LS phases, and a phase diagram was constructed.

High-pressure Raman spectra and ruby fluorescence spectra were acquired using a LabramHR (Horiba Jobin-Yvon) microspectrometer. The orientation of the sample crystal was kept constant during each measurement due to a significant dependence of peak intensities on orientation of the crystal with respect to the polarization direction of the incident laser beam. A HeNe laser $(632.8 \mathrm{~nm})$ was used as an excitation source; the beam was focused on a spot of approximately $2 \mu \mathrm{m}$ via a $50 \times$ long-working-distance objective. The Rayleigh scattering was removed by a holographic notch filter, and the Raman spectra of the sample were recorded between 2000-2220 $\mathrm{cm}^{-1}$, using a 1800 grooves/mm grating with a spectral resolution of $\sim 1 \mathrm{~cm}^{-1}$. The ruby fluorescence spectra were recorded between 687 and $700 \mathrm{~nm}$, using the same setup.

Magnetic susceptibility measurements at high pressure were carried out under a magnetic field of $1 \mathrm{~T}$ with heating and cooling rates of $0.5 \mathrm{~K} / \mathrm{min}$ using a clamp-type cell made from hardened $\mathrm{CuBe}$ as previously described. ${ }^{41} \mathrm{~A}$ pressure-transmitting oil (Alcatel-500) was used to ensure a hydrostatic environment at the sample. The pressure inside the cell was inferred from the temperature shift of the lead and/or tin superconducting transition, both of which are determined below $10 \mathrm{~K} .^{42}$ These are the values quoted in the text, although the pressure inside the cell increases with temperature, at $300 \mathrm{~K}$, reaching an estimated 1-2 kbar greater than that
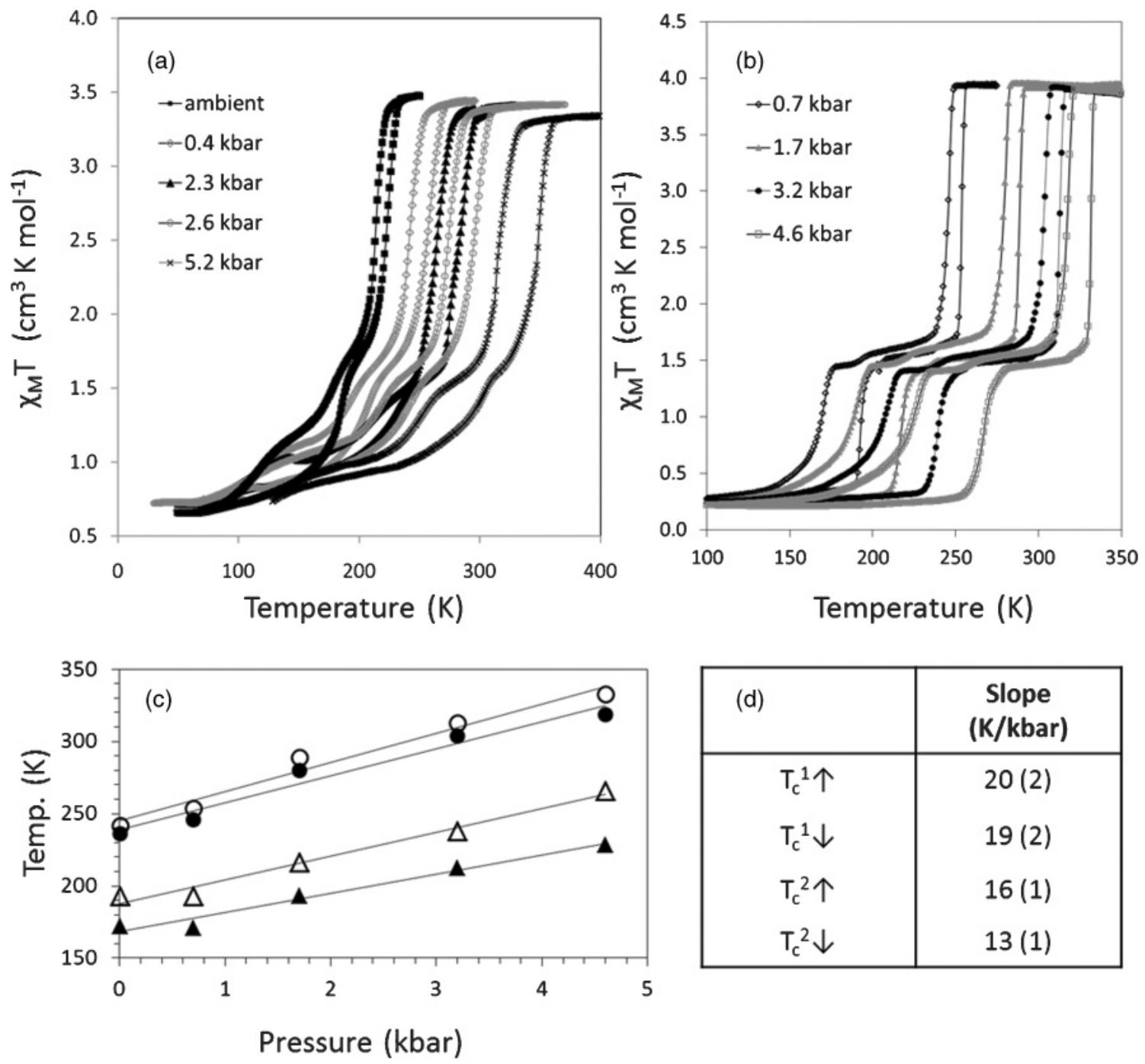

\begin{tabular}{|c|c|}
\hline (d) & $\begin{array}{l}\text { Slope } \\
\text { (K/kbar) }\end{array}$ \\
\hline$T_{c}^{1} \uparrow$ & $20(2)$ \\
\hline$T_{c}^{1} \downarrow$ & $19(2)$ \\
\hline$T_{c}^{2} \uparrow$ & $16(1)$ \\
\hline$T_{c}^{2} \downarrow$ & $13(1)$ \\
\hline
\end{tabular}

FIG. 2. Pressure dependence of $\chi_{M} T$ vs $T$ curves for (a) a precipitated powder and (b) a slowly crystallized sample of $\mathbf{1}$. Solid lines are shown as a guide for the eye. The transition temperatures as a function of pressure are shown in (c). Filled circles indicate the transition temperature on cooling for the high-temperature step $\left(\mathrm{T}_{\mathrm{c}}{ }^{1} \downarrow\right)$; filled triangles are those of the second step $\left(\mathrm{T}_{\mathrm{c}}{ }^{2} \downarrow\right)$. Equivalent open points indicate the respective transition temperatures on warming $\left(\mathrm{T}_{\mathrm{c}}{ }^{1} \uparrow\right.$ and $\left.\mathrm{T}_{\mathrm{c}}{ }^{2} \uparrow\right)$. The straight lines give a linear fit of each set of points, the slopes of which are presented in (d) together with the error bars (in parenthesis). 
recorded at low temperature. Analysis of the high-pressure magnetic data was complicated by a strong diamagnetic contribution to the magnetization from the pressure cell and pressure-transmitting oil, as well as the inability to obtain an accurate value for the mass of the sample. Although suitable estimates of the diamagnetic contribution and mass of the sample were used to correct the data, absolute values of $\chi_{\mathrm{M}} \mathrm{T}$ should be treated with caution.

\section{MAGNETOMETRY}

Variable temperature magnetic susceptibility data were recorded at various fixed pressures between ambient pressure and $5.2 \mathrm{kbar}$ on crude powder samples of $\mathbf{1},{ }^{19}$ as well as on samples slowly recrystallized by liquid-liquid diffusion of methanol into Dimethylformamide (DMF) ${ }^{18}$ between ambient pressure and 4.6 kbar (Figs. 2(a) and 2(b)). For the powder sample, it is clear that while the hysteresis of the hightemperature step widens significantly on increasing pressure, the low-temperature transition becomes very distorted and nontrivial to rationalize. An even greater distortion of this second step was seen in the previous high-pressure magnetic study of this complex ${ }^{19}$ (which also used a powdered form of 1) to such an extent that this low-temperature step was largely suppressed at elevated pressures. As this is not the case for a collection of single crystals, such distortion is attributed to the inhomogeneity of the precipitated powder. This might include impurities, strain, or grain size distribution. A difference in magnetic behavior between the powdered and recrystallized forms of $\mathbf{1}$ at ambient pressure has also been reported, ${ }^{19}$ that the effect of pressure serves to amplify these differences is clear from the comparison of the curves presented in Fig. 2. Detailed analysis of the structural differences between the powdered and recrystallized forms and the influence this has on the magnetic behavior under pressure is beyond the scope of this work, thus all subsequent Raman spectroscopic and x-ray diffraction measurements were performed on single crystals of $\mathbf{1}$.

For the recrystallized sample of $\mathbf{1}$, both the $\mathrm{HS} \rightarrow \mathrm{IP}$ and $\mathrm{IP} \rightarrow$ LS steps remain characteristically abrupt up to $4.6 \mathrm{kbar}$; furthermore, the hysteresis loops of both steps widen as the pressure increases. The variation of the transition temperature on heating and cooling for both steps of the transition are plotted as a function of pressure for the crystalline sample in Fig. 2(c). Linear fits to these data yield slopes $[\Delta T / \Delta P$, Fig. 2(d)] that reveal both transition temperatures are increased by pressure as expected. These slopes are directly comparable with the Clapeyron slopes $(\Delta \mathrm{V} / \Delta \mathrm{S}=16 \pm 2$ and $14 \pm$ $2 \mathrm{~K} / \mathrm{kbar}$ for the first and second step of the transition, respectively ${ }^{18}$ ) and fall in the range of previously reported values for mononuclear iron(II) SCO materials. ${ }^{22,43}$

Clearly both hystereses broaden as pressure increases, which is contrary to the expectation from the standard theoretical models. ${ }^{23}$ Such theories do not, however, consider a change in crystallographic symmetry between the HS and LS states or indeed that the lattice itself can be pressure dependant. Previous experimental observation of widening of the hysteresis loop on increasing pressure include $\left[\mathrm{Fe}(\mathrm{phy})_{2}\right]\left(\mathrm{ClO}_{4}\right)^{44,45}$ and $\left[\mathrm{Fe}(\mathrm{PM}-\mathrm{Bia})_{2}(\mathrm{NCS})_{2}\right]{ }^{46,47}$ In the former, a crystallographic phase transition between the HS and LS states at ambient pressure was demonstrated by powder diffraction. ${ }^{48}$ In the case of $\left[\mathrm{Fe}(\mathrm{PM}-\mathrm{Bia})_{2}(\mathrm{NCS})_{2}\right]$, at ambient pressure there is no difference in crystallographic symmetry between HS and LS states; however, the possibility of a second (pressure-induced) phase of this material that does show such a crystallographic transition between spin states has been postulated to explain the increase in width of the hysteresis above 6 kbar. $^{46,47}$ An alternative explanation for the increase in hysteresis width is that there is an increase in the particle size distribution, for example, in the case of [ $\left.\mathrm{Fe}(\text { bipy })_{2}(\mathrm{NCS})_{2}\right]$, where the widening and flattening of the hysteresis is attributed to the mechanical grinding of the sample. ${ }^{44}$ Such an effect can be ruled out in the case of $\mathbf{1}$, as the hysteresis broadening is reversible, with hysteresis widths of $6 \mathrm{~K}$ and $21 \mathrm{~K}$ for the $\mathrm{HS} \rightarrow \mathrm{IP}$ and $\mathrm{IP} \rightarrow \mathrm{LS}$ transitions, respectively, after decompression to atmospheric pressure from $4.6 \mathrm{kbar}$, as shown in the electronic supplementary information (Fig. S1). ${ }^{32,33}$

\section{RAMAN SPECTROSCOPY}

The thermally induced HS, IP, and LS phases have been thoroughly characterized at ambient pressure using Raman spectroscopy and characteristic modes have been assigned to
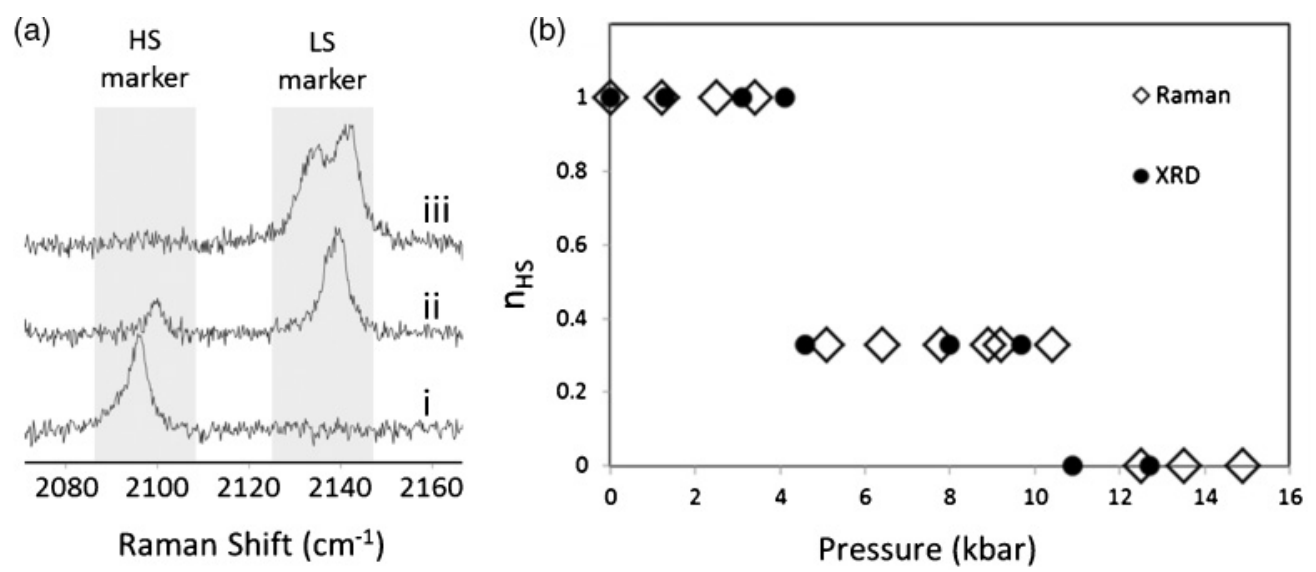

FIG. 3. (a) Raman spectra of compound 1 in the cyano-stretching region at (i) 2.5 , (ii) 7.8 , and (iii) 13.5 kbar, highlighting the characteristic peaks for the HS and LS molecular spin states. (b) Evolution of the HS fraction in a single crystal of 1 under compression obtained from high-pressure Raman and x-ray diffraction (XRD) data. 
TABLE I. Unit cell lattice parameters (and their standard deviations) of $\mathbf{1}$ at different pressures.

\begin{tabular}{|c|c|c|c|c|c|c|c|c|}
\hline Pressure / kbar & ambient (in DAC) & 1.3 & 2.9 & 4.1 & 8.0 & 9.7 & 12.7 & Depressurized \\
\hline Space group & $C 2 / c$ & $C 2 / c$ & $C 2 / c$ & $C 2 / c$ & $C 2 / c$ & $C 2 / c$ & $P \overline{1}$ & $C 2 / c$ \\
\hline$a / \AA$ & $15.90(4)$ & $16.04(5)$ & $15.86(3)$ & $15.73(7)$ & $15.627(7)$ & $15.54(2)$ & $9.34(3)$ & $15.99(2)$ \\
\hline$b / \AA$ & $11.21(4)$ & $10.90(4)$ & $11.11(3)$ & $10.94(6)$ & $10.777(9)$ & $10.70(2)$ & $9.36(4)$ & $11.08(2)$ \\
\hline$c / \AA$ & $14.40(2)$ & $14.36(2)$ & $14.22(1)$ & $14.06(3)$ & $41.675(7)$ & $41.49(1)$ & $12.90(3)$ & $14.376(9)$ \\
\hline$\alpha /^{\circ}$ & & & & & & & $93.2(5)$ & \\
\hline$\beta /{ }^{\circ}$ & $117.7(2)$ & $117.3(2)$ & $117.8(2)$ & $117.5(4)$ & $116.83(2)$ & $116.82(4)$ & $105.5(3)$ & 117.3(1) \\
\hline$\gamma /^{\circ}$ & & & & & & & $110.9(4)$ & \\
\hline Volume $/ \AA^{3}$ & $2271(6)$ & $2231(7)$ & $2216(6)$ & $2145(13)$ & $6263(4)$ & $6156(9)$ & $1000(6)$ & $2265(4)$ \\
\hline Normalized volume $/ \AA^{3}$ & 2271 & 2231 & 2216 & 2145 & 2088 & 2052 & 2000 & 2265 \\
\hline Assigned phase & HS & HS & HS & HS & IP & IP & LS & HS \\
\hline
\end{tabular}

HS and LS species within the cyano-stretching $\left(v_{\mathrm{CN}}\right)$ region of the spectrum. A large frequency shift was observed between the HS $\left(2098 \mathrm{~cm}^{-1}\right)$ and LS $\left(2138 \mathrm{~cm}^{-1}\right)$ forms, which could be explained by the concomitant effect of weaker $\sigma$ donation and stronger $\pi$ backdonation on the $\mathrm{CN}$ bond strength in the HS $\left({ }^{5} \mathrm{~T}_{2}, \mathrm{t}_{2 \mathrm{~g}}{ }^{4} \mathrm{e}_{\mathrm{g}}{ }^{2}\right)$ configuration when compared to the LS $\left({ }^{1} \mathrm{~A}_{1}, \mathrm{t}_{2 \mathrm{~g}}{ }^{6} \mathrm{e}_{\mathrm{g}}{ }^{0}\right)$ form. In the low-temperature LS phase, a well-resolved splitting of this mode $\left(2134\right.$ and $\left.2139 \mathrm{~cm}^{-1}\right)$ provides a clear spectral signature of the phase, compared to the IP. As shown in Fig. 3(a), very similar spectral changes can be observed under compression at room temperature, thus enabling the assignment of HS, IP, and LS phases to the sample at each pressure by analogy to the thermal evolution at ambient pressure. The ratio of the integrated area of the $v_{\mathrm{CN}}$ modes has previously been used to reproduce the thermal two-step transition behavior. ${ }^{31}$ In this study a ratio of $\sim 25 \%$ was shown to correspond to the intermediate phase, characterized by the HS-LS-LS motif. The variation of the HS fraction, $\mathrm{n}_{\mathrm{HS}}$, as a function of pressure as deduced from the high-pressure Raman data is given in Fig. 3(b). Between atmospheric pressure and $3.4 \mathrm{kbar}$, the complex in is the high-spin state. On increasing the pressure, a plateau can be clearly observed between 5.1 and $10.4 \mathrm{kbar}$, corresponding to the intermediate phase. Above $12.5 \mathrm{kbar}$ only the LS state is present. At no pressure is there any indication of the presence of more than one phase revealing that the $\mathrm{HS} \rightarrow \mathrm{IP}$ and $\mathrm{IP} \rightarrow \mathrm{LS}$ transitions are discontinuous (within less than $0.5 \mathrm{kbar}$ ), even as a function of pressure. If one considers also the data obtained from the $\mathrm{x}$-ray diffraction experiments (vide infra) the $\mathrm{n}_{\mathrm{HS}}(\mathrm{P})$ curve in Fig. 3(b) is indicative of two first-order transitions, by analogy with the thermal phase transitions. Due to experimental limitations, it was not possible to measure the effect of decreasing pressure on the transition, and thus the possible presence of piezo-hysteresis could not be directly measured. However, extrapolation of the linear fits of the transition temperatures as a function of pressure shown in Fig. 2 indicates that hysteresis loops with widths of 0.6 and 2.9 kbar would be expected at $295 \mathrm{~K}$ for the $\mathrm{HS} \rightarrow \mathrm{IP}$ and IP $\rightarrow$ LS transitions, respectively.

\section{X-RAY DIFFRACTION}

In an effort to determine whether this pressure-induced stepped spin transition is accompanied by a crystallographic symmetry breaking analogous to that observed during the thermal transition or by other pressure-induced structural changes, high-pressure, single-crystal x-ray diffraction experiments were performed. Diffraction techniques can provide important information in the study of SCO. For example, the analysis of bond lengths and angles at the iron center can unambiguously allow assignment of spin state, and in the case of an intermediate state between pure HS and LS, it provides information regarding the distribution of domains or long-range order of different sites.

Cell parameters for $\mathbf{1}$, determined from the sample inside the DAC at seven pressures between ambient and $12.7 \mathrm{kbar}$, are

TABLE II. Comparison of selected bond distances $(\AA)$ and angles $\left(^{\circ}\right)$ for the temperature- (from Ref. 18) and pressure-induced intermediate phases.

\begin{tabular}{|c|c|c|c|c|c|}
\hline $\mathrm{Fe} 2$ & IP (190 K) & IP (4.6 kbar) & $\mathrm{Fe} 1$ & IP (190 K) & IP (4.6 kbar) \\
\hline Fe2-N9 & $2.135(2)$ & $2.152(4)$ & $\mathrm{Fe} 1-\mathrm{N} 1$ & $1.980(2)$ & $2.000(3)$ \\
\hline Fe2-N11 & $2.131(2)$ & $2.139(4)$ & $\mathrm{Fe} 1-\mathrm{N} 3$ & $1.942(2)$ & $1.938(4)$ \\
\hline \multirow[t]{4}{*}{$\mathrm{Fe} 2-\mathrm{N} 12$} & $2.149(3)$ & $2.162(8)$ & Fe1-N4 & $1.932(2)$ & $1.926(8)$ \\
\hline & & & Fe1-N5 & $1.942(2)$ & $1.939(4)$ \\
\hline & & & Fe1-N7 & $1.983(2)$ & $1.998(4)$ \\
\hline & & & Fe1-N8 & $1.936(2)$ & 1.937(8) \\
\hline N9-Fe2-N11 & $85.76(8)$ & $85.69(2)$ & $\mathrm{N} 1-\mathrm{Fe} 1-\mathrm{N} 3$ & $91.41(9)$ & $91.0(2)$ \\
\hline N9-Fe2-N11 a & $77.47(12)$ & $76.6(3)$ & N3-Fe1-N5 & $82.46(9)$ & $82.0(2)$ \\
\hline $\mathrm{N} 11^{\mathrm{a}}-\mathrm{Fe} 2-\mathrm{N} 9^{\mathrm{a}}$ & $85.76(8)$ & $85.69(2)$ & N5-Fe-N7 & $92.13(9)$ & $93.0(2)$ \\
\hline N9 ${ }^{\mathrm{a}}-\mathrm{Fe} 2-\mathrm{N} 9$ & $113.44(12)$ & $114.7(3)$ & N7-Fe1-N1 & $95.83(9)$ & $96.4(2)$ \\
\hline $\mathrm{N} 12^{\mathrm{a}}-\mathrm{Fe} 2-\mathrm{N} 12$ & $167.70(13)$ & $165.7(5)$ & N4-Fe1-N8 & 177.30(9) & 177.1(3) \\
\hline
\end{tabular}

a Symmetry codes: 1-x, y, $\frac{1}{2}-\mathrm{z}$. 

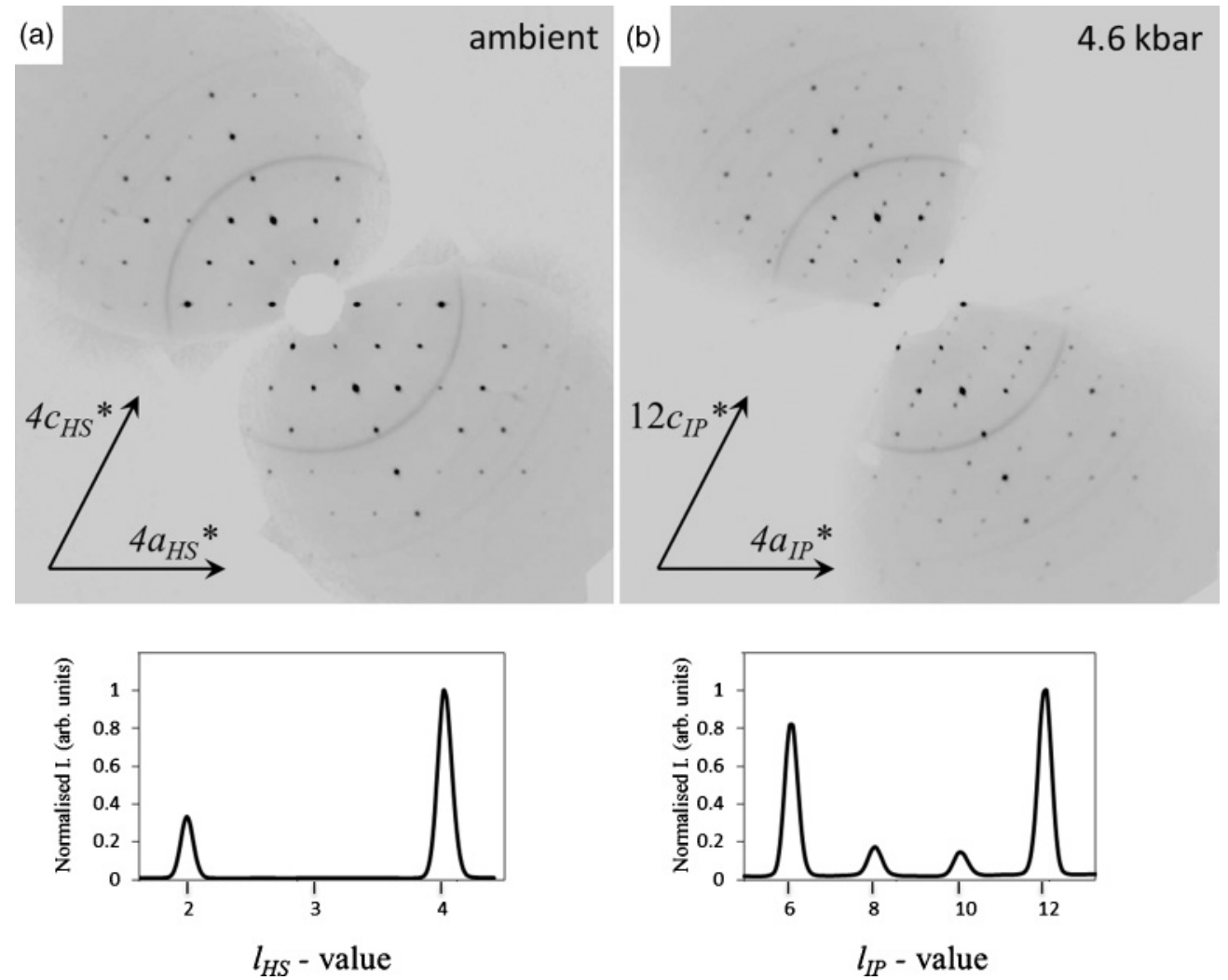

FIG. 4. Pseudoprecession images of the reciprocal lattice in the (h0l) plane for $\mathbf{1}$ at (a) ambient pressure (inside the DAC) and (b) $4.6 \mathrm{kbar}$, reconstructed from the collected diffraction images. For illustrative purposes a plot of normalized intensity along $(-6,0, l)$ for values of $l$ between 2 and 4 in the ambient pressure setting is also shown for each image.

presented in Table I. A tripling of the $c$-axis was observed at 8.0 and $9.7 \mathrm{kbar}$, demonstrated by the appearance of supercell reflections at $(h, k, l \pm 1 / 3)$ relative to the lattice at ambient pressure. At $12.7 \mathrm{kbar}$ the diffraction pattern is complicated by the presence of multiple peaks, which cannot be assigned to one lattice. This same feature was observed in the LS phase at low temperatures and was attributed to twinning occurring with the lowering of symmetry from $2 / \mathrm{m}$ to $\overline{1}$ during the $\mathrm{IP} \rightarrow \mathrm{LS}$ transition. The twin law is such that the peaks overlap strongly at low angle, while they are well resolved at higher $\theta$ values. As the high-pressure experiments were carried out at ambient temperature, diffraction from the pressure-induced LS species is not observed to as high an angle as in the low-temperature LS state. Consequently, the peaks are not well resolved, and although it was possible to index one component of the diffraction pattern to a comparable primitive cell as the low-temperature LS state, additional components could not. The pressure-induced structural transformation is reversible, as evidenced by the cell parameters measured after decompression of the sample from 12.7 kbar (Table I). Hence, the multiple peaks observed in the diffraction pattern at 12.7 kbar are not indicative of disintegration of the crystal but are rather significant of the structural and spin transitions. It is thus concluded that the pressure-induced LS state is structurally similar to that observed at low temperature, which is also supported by the similarity of Raman spectra of the pressure- and temperature-induced LS phases (see electronic supplementary information).
X-ray diffraction data were also collected at ambient pressure (inside the DAC), 4.6 and 10.9 kbar with a view to structural refinement of the pressure-induced IP and LS phases. Selected bond distances and angles from the refinement at ambient pressure and $4.6 \mathrm{kbar}$ are presented in Table II, along with those obtained by Bonnet et al. ${ }^{18}$ from their variable temperature study. The LS phase was identified at 10.9 kbar, but it was not possible to refine the structure due to the previously mentioned structural complications associated with this phase. The $c$-axis tripling on passing from $\mathrm{HS} \rightarrow$ IP phase may be clearly seen in the diffraction pattern; pseudoprecession images of the (h0l) plane reconstructed from the diffraction images are shown in Fig. 4. The ambient pressure structure (inside the DAC) shows no significant differences from that previously published, other than the expected reduction in quality of the refinement statistics, attributable to the restrictions of the DAC as described previously. The structure at $4.6 \mathrm{kbar}$ is isostructural with the intermediate phase determined at ambient pressure (190 $\mathrm{K}) .{ }^{18}$ Detailed analysis of the crystal structure at $4.6 \mathrm{kbar}$ is given in the electronic supplementary information, along with comparison to the isostructural intermediate phase structure from the ambient pressure study at $190 \mathrm{~K}$.

The spin state was assigned to each $\mathrm{Fe}^{\mathrm{II}}$ center by considering the volume of the octahedron defined by the six ligating nitrogen atoms $\left(\mathrm{V}_{\mathrm{p}}\right)$. This measure allows for a simple comparison of the coordination geometry (both bond lengths and angles) at different iron centers. Typically this volume is 


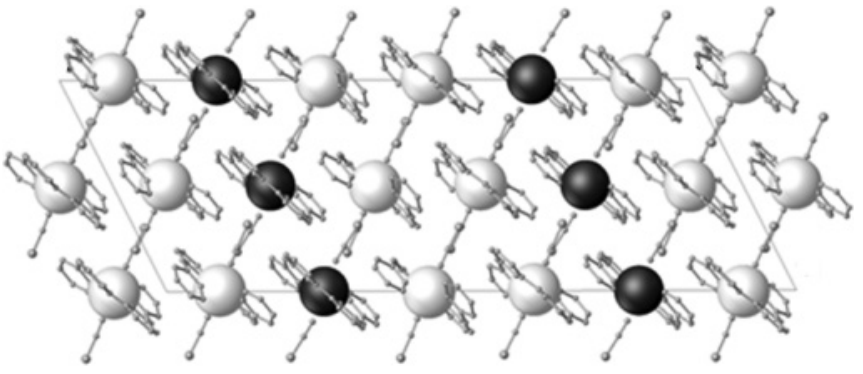

FIG. 5. Extended structure of $\mathbf{1}$ at $4.6 \mathrm{kbar}$ perpendicular to the $a c$-plane revealing the long-range [HS-LS-LS] order of this pressureinduced intermediate phase. Iron atoms are shown as large spheres, for illustrative purposes. LS Fe atoms are colored white, and HS Fe atoms are colored black. All other atoms are shown as small grey spheres. Hydrogen atoms have been omitted for clarity.

approximately $25 \%$ larger in the HS rather than LS state. ${ }^{25}$ In the previous study, the value for $\mathrm{V}_{\mathrm{p}}$ in the HS phase was $12.5 \AA^{3}$, and $\mathrm{V}_{\mathrm{p}}(\mathrm{HS})$ and $\mathrm{V}_{\mathrm{p}}(\mathrm{LS})$ in the intermediate phase were 12.4 and $9.8 \AA^{3}$, respectively. ${ }^{18}$ At $4.6 \mathrm{kbar}$ there are two distinct iron centers, one of which resides on a twofold rotation axis. The iron atom $\mathrm{Fe} 2$, which is located on the special position, has $\mathrm{V}_{\mathrm{p}}=12.4(1) \AA^{3}$ (HS state) and that which is located on a general position has $V_{p}=9.8(1) \AA^{3}$ (LS state). The single-crystal $\mathrm{X}$-ray diffraction experiment therefore reveals that the ratio of HS to LS centers in the structure at $4.6 \mathrm{kbar}$ is $1: 2$. Furthermore there is long-range order of the [HS-LS-LS] motif throughout the crystal, as shown in Fig. 5.

The pressure-temperature phase diagram of $\mathbf{1}$, derived from diffraction data from the previous ambient pressure study ${ }^{33}$ and data collected at high pressure on beamline ID27 at the ESRF is shown in Fig. 6. Rather than the disappearance of the intermediate phase with pressure, as in the case of [Fe(2pic) $\left.{ }_{3}\right] \mathrm{Cl}_{2} \cdot \mathrm{EtOH}^{23}$ and $\mathrm{Fe}(3 \text {-methylpyridine })_{2}\left[\mathrm{Ni}(\mathrm{CN})_{4}\right],{ }^{41}$ it is clear that in 1, the IP may be observed across a large range of temperature and pressure. The linear fit of the transition temperatures of both transitions (obtained from magnetic

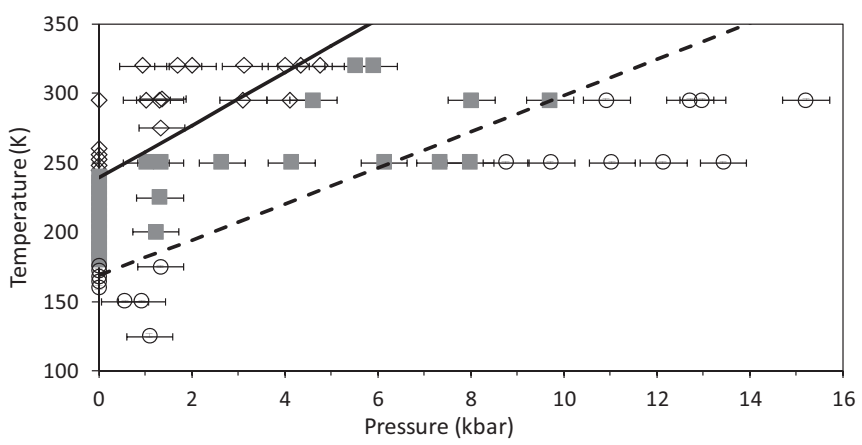

FIG. 6. Pressure-temperature phase diagram of 1. All discrete points are determined from diffraction data. Ambient pressure values are as determined in Ref. 33. Open diamonds represent the HS phase, grey squares the IP, and open circles the LS phase. The solid line shows the linear fit (slope $=19 \mathrm{~K} / \mathrm{kbar}$ ) of the HS $\rightarrow$ IP transition temperature on cooling as a function of pressure from the magnetic measurements presented in Fig. 2(c). The dashed line shows that of the $\mathrm{IP} \rightarrow \mathrm{LS}$ transition (slope $=13 \mathrm{~K} / \mathrm{kbar}$ ). measurements) is also indicated in the figure, and there is good agreement between the two methods. Slight discrepancies between the results obtained from diffraction methods and the magnetic study are attributed to the experimental error involved with pressure determination for both techniques, particularly the intrinsic difference in pressure inside the magnetic cell between the temperature at which the pressure is measured and the transition temperature, as described in the experimental section.

\section{DISCUSSION AND CONCLUSIONS}

To a first approximation, the effects of pressure on the SCO phenomenon are simply related to the volume change of the unit cell accompanying the SCO and the associated change of the HS-LS energy gap by the work term $(P \Delta V)$. From a thermodynamic point of view, these effects can be described by the well-known equation of state: ${ }^{46}$

$$
T=\frac{\Delta H_{\mathrm{HL}}+P \Delta V_{\mathrm{HL}}-2 n_{\mathrm{HS}} \Gamma}{k_{B} \ln \left(\frac{1-n_{\mathrm{HS}}}{n_{\mathrm{HS}}}\right)+\Delta S_{\mathrm{HL}}},
$$

where $\Delta \mathrm{H}_{\mathrm{HL}}, \Delta \mathrm{S}_{\mathrm{HL}}$, and $\Delta \mathrm{V}_{\mathrm{HL}}$ stand for the enthalpy, entropy, and volume changes accompanying the spin-state change, respectively, and $\Gamma$ is a phenomenological (mean-field) interaction constant. Across a relatively narrow range of $T$ - $P$, these parameters can be considered independent of temperature and pressure. Hence, with increasing pressure a linear shift of the transition to higher temperatures and a decrease of the hysteresis width (or the slope of the transition curve) can be predicted. However, the experimental observations do not fit these expectations in many cases. Indeed, in past decades a variety of SCO behaviors under applied pressure have been reported in the literature, including "anomalous" phenomena such as increasing or nonmonotonously changing thermal hysteresis widths, a pressure-induced shift of the thermal transition curve without a change of its shape, stabilization of the HS phase, or a nonlinear shift of $T_{c}{ }^{22,47}$ The reason behind this variety of behavior is that pressure is coupled to the order parameter by various mechanisms, which remain largely unexplored up to now. For example, coupling between structural phase transformations and SCO is often evoked in the literature, but the interpretation of the experimental observations has been at times compromised by the relatively poor and incomplete spectral and structural information provided by the detection methods or due to the experimental difficulties related to the need for hydrostatic conditions at low temperatures.

It is thus important to underline that the combined complimentary techniques used in the present study of $\left.\left[\mathrm{Fe}^{\mathrm{II}} \text { (bapbpy)(NCS }\right)_{2}\right]$ allowed us to map the pressuretemperature phase diagram with unprecedented detail. The influence of pressure on the thermal two-step transition and the hysteresis of both steps were found to be rather unexpected in this compound. In particular, one shall note that the thermal hysteresis width of both transitions increases with pressure, which is quite unusual. The presence of a two-step spin transition under pressure at ambient temperature was also revealed in conjunction with a crystallographic symmetry breaking that accompanies both (first-order) steps as a function 
of pressure. Further diffraction experiments across a range of both temperature and pressure confirmed that the ordered HS-LS-LS intermediate phase is stable across a wide region of pressure and temperature values. In other words, the same $\mathrm{HS} \leftrightarrow \mathrm{IP} \leftrightarrow \mathrm{LS}$ sequence of spin and crystallographic transitions has been observed within the whole investigated $P-T$ space. This thorough description of pressure-induced magnetostructural correlations in $\mathbf{1}$ provides a reliable platform for testing the validity of different models of SCO proposed in the literature.

We finish with a few remarks on the theoretical prospects. In order to reproduce the experimentally observed two-step $(1: 2)$ thermal transition in $\mathbf{1}$, we previously proposed the use of an extension to the "standard" mean-field approximation Isinglike model ${ }^{49}$ with three interacting sublattices. ${ }^{19}$ Indeed, when applied to the thermal evolution of the high-spin fraction $\left(\mathrm{n}_{\mathrm{HS}}\right)$ at atmospheric pressure, this model closely reproduces the experimentally observed equilibrium temperatures, hysteresis loop widths as well as the plateau around $\mathrm{n}_{\mathrm{HS}}=1 / 3$ (Fig. 1). However, in the $(p, T)$ plane this model cannot describe the experimentally observed phase diagram — in particular the hightemperature piezo-hysteresis loops (see details in the electronic supporting information). Indeed, in its mean-field form, the Ising-like model is equivalent to the thermodynamic models. On the other hand, the microscopic origin of the Ising-like model provides a flexibility, which allows the consideration of correlations between molecules with the help of numerical ${ }^{50}$ or analytical ${ }^{51,52}$ methods. Hence this model could be extended to provide insights into the electron-phonon coupling involved in two-step spin transitions. ${ }^{53,54}$ Using anharmonic interaction potentials, ${ }^{55}$ one might also access the thermoelastic coefficients of the system, which can then be compared to experimental x-ray diffraction data. Another approach-the so-called elasticity theory-was proposed by Spiering et al. for modeling of various SCO behaviors-including stepped transition curves. ${ }^{44,56}$ Monte Carlo techniques have been also implemented with good fit to the experimental two-step $\mathrm{n}_{\mathrm{HS}}(\mathrm{T})$ curves in the isobaric regime. ${ }^{57}$ These authors were also the first to predict the establishment of a "chessboard structure" in the IP. ${ }^{58}$ The interesting feature of their model is that it takes into account explicitly the temperature and pressure dependence of the relevant properties of the crystal lattice. For example, through the volume dependence of the bulk modulus, it was possible to simulate the anomalous increase of the hysteresis width. Finally, it should be noted that Landau theory has been also applied to the problem of stepped spin transitions and extended to include the possibility of crystallographic symmetry breaking with long-range order of HS and LS sites at the plateau in both the isothermal and isobaric regimes. ${ }^{59}$ Although this model is based solely on symmetry-based arguments, it was used to describe rather satisfactorily the symmetry breaking observed in $\left[\mathrm{Fe}(2 \text {-pic })_{3}\right] \mathrm{Cl}_{2} \cdot \mathrm{EtOH}^{9}$ and the increasing width of the thermal hysteresis of general systems, showing first-order transitions have also been discussed by considering the coupling of elastic strain and the order parameter. $^{60}$

\section{ACKNOWLEDGMENTS}

Financial support was provided by the ANR Project SCOOP (ANR-08-JCJC-0049-01). Marc Nardone (LNCMI, Toulouse) and Lionel Rechignat (LCC, Toulouse) are acknowledged for their help with the high-pressure magnetic cell. Leiden University is acknowledged for financial support to S.B.
*Corresponding author: gabor.molnar@1cc-toulouse.fr

${ }^{1}$ Y. Garcia, O. Kahn, L. Rabardel, B. Chansou, L. Salmon, and J.-P. Tuchagues, Inorg. Chem. 38, 4663 (1999).

${ }^{2}$ J. A. Real, H. Bolvin, A. Bousseksou, A. Dworkin, O. Kahn, F. Varret, and J. Zarembowitch, J. Am. Chem. Soc. 114, 4650 (1992).

${ }^{3}$ J. A. Real, A. B. Gaspar, M. C. Muñoz, P. Gütlich, V. Ksenofontov, and H. Spiering, Top. Curr. Chem. 233, 167 (2004).

${ }^{4}$ V. Ksenofontov, A. B. Gaspar, V. Niel, S. Reiman, J. A. Real, and P. Gütlich, Chem. Eur. J. 10, 1291 (2004).

${ }^{5}$ S. M. Neville, B. A. Leita, G. J. Halder, C. J. Kepert, B. Moubaraki, J.-F. Létard, and K. S. Murray, Chem. Eur. J. 14, 10123 (2008).

${ }^{6}$ G. J. Halder, K. W. Chapman, S. M. Neville, B. Moubaraki, K. S. Murray, J.-F. Létard, and C. J. Kepert, J. Am. Chem. Soc. 130, 17552 (2008).

${ }^{7}$ M. Nihei, M. Ui, M. Yokota, L. Han, A. Maeda, H. Kishida, H. Okamoto, and H. Oshio, Angew. Chem. Int. Ed. 44, 6484 (2005).

${ }^{8}$ A. Kaiba, H. J. Shepherd, D. Fedaoui, P. Rosa, A. E. Goeta, N. Rebbani, J.-F. Létard, and P. Guionneau, Dalton Trans. 39, 2910 (2010).

${ }^{9}$ D. Chernyshov, M. Hostettler, K. W. Törnroos, and H.-B. Bürgi, Angew. Chem. Int. Ed. 42, 3825 (2003).
${ }^{10}$ N. Bréfuel, E. Collet, H. Watanabe, M. Kojima, N. Matsumoto, L. Toupet, K. Tanaka, and J.-P. Tuchagues, Chem. Eur. J. 16, 14060 (2010).

${ }^{11}$ K. Nakano, S. Kawata, K. Yoneda, A. Fuyuhiro, T. Yagi, S. Nasu, S. Morimoto, and S. Kaizaki, Chem. Commun. 2892 (2004).

${ }^{12}$ K. W. Törnroos, M. Hostettler, D. Chernyshov, B. Vangdal, and H.-B. Bürgi, Chem. Eur. J. 12, 6207 (2006)

${ }^{13}$ M. Yamada, H. Hagiwara, H. Torigoe, N. Matsumoto, M. Kojima, F. Dahan, J.-P. Tuchagues, N. Re, and S. Iijima, Chem. Eur. J. 12, 4536 (2006).

${ }^{14}$ N. Bréfuel, H. Watanabe, L. Toupet, J. Come, N. Matsumoto, E. Collet, K. Tanaka, and J.-P. Tuchagues, Angew. Chem. Int. Ed. 48, 9304 (2009).

${ }^{15}$ M. Griffin, S. Shakespeare, H. J. Shepherd, C. J. Harding, J.-F. Létard, C. Desplanches, A. E. Goeta, J. A. K. Howard, A. K. Powell, V. Mereacre, Y. Garcia, A. D. Naik, H. Müller-Bunz, and G. G. Morgan, Angew. Chem. Int. Ed. 50, 896 (2011).

${ }^{16}$ M. Nihei, H. Tahira, N. Takahashi, Y. Otake, Y. Yamamura, Kazuya Saito, and H. Oshio, J. Am. Chem. Soc. 132, 3553 (2010).

${ }^{17}$ M. Buron-Le Cointe, N. Ould Moussa, E. Trzop, A. Moréac, G. Molnár, L. Toupet, A. Bousseksou, J.-F. Létard, and G. S. Matouzenko, Phys. Rev. B 82, 214106 (2010). 
${ }^{18}$ S. Bonnet, M. A. Siegler, J. Sánchez Costa, G. Molnár, A. Bousseksou, A. L. Spek, P. Gamez, and J. Reedijk, Chem. Commun. 5619 (2008).

${ }^{19}$ S. Bonnet, G. Molnár, J. Sánchez Costa, M. A. Siegler, A. L. Spek, A. Bousseksou, W.-T. Fu, P. Gamez, and J. Reedijk, Chem. Mater. 21, 1123 (2009).

${ }^{20}$ T. Sato, K. Nishi, S. Iijima, M. Kojima, and N. Matsumoto, Inorg. Chem. 48, 7211 (2009).

${ }^{21}$ A. Bousseksou, F. Varret, and J. Nasser, J. Phys. I (France) 3, 1463 (1993).

${ }^{22}$ V. Ksenofontov, A. B. Gaspar, and P. Gütlich, Top. Curr. Chem. 235, 23 (2004).

${ }^{23}$ C. P. Köhler, R. Jakobi, E. Meissner, L. Wiehl, H. Spiering, and P. Gütlich, J. Phys. Chem. Solids 51, 239 (1990).

${ }^{24}$ P. Gütlich, A. Hauser, and H. Spiering, Angew. Chem. Int. Ed. Engl. 33, 2024 (1994).

${ }^{25}$ P. Guionneau, M. Marchivie, G. Bravic, J.-F. Létard, and D. Chasseau, Top. Curr. Chem. 234, 97 (2004).

${ }^{26} \mathrm{P}$. Gütlich, A. B. Gaspar, Y. Garcia, and V. Ksenofontov, C. R. Chimie 10, 21 (2007).

${ }^{27}$ P. Guionneau, C. Brigouleix, Y. Barrans, A. E. Goeta, J.-F. Létard, J. A. K. Howard, J. Gaultier, and D. Chasseau, C. R. Acad. Sci. IIC 4, 161 (2001).

${ }^{28}$ T. Granier, B. Gallois, J. Gaultier, J. A. Real, and J. Zarembowitch, Inorg. Chem. 32, 5305 (1993).

${ }^{29}$ P. Guionneau, M. Marchivie, Y. Garcia, J. A. K. Howard, and D. Chasseau, Phys. Rev. B 72, 214408 (2005).

${ }^{30}$ V. Legrand, F. Le Gac, P. Guionneau, and J.-F. Létard, J. Appl. Crystallogr. 41, 637 (2008).

${ }^{31}$ S. Bedoui, G. Molnár, S. Bonnet, C. Quintero, H. J. Shepherd, W. Nicolazzi, L. Salmon, and A. Bousseksou, Chem. Phys. Lett. 499, 94 (2010).

${ }^{32} \mathrm{See}$ Supplemental Material at http://link.aps.org/supplemental/ 10.1103/PhysRevB.84.144107 for additional magnetic, crystallographic and Raman spectral data as well as for details on the Ising-like model with three sublattices.

${ }^{33}$ Detailed structural analysis and cell parameter evolution as a function of temperature are given in the electronic supplementary material of S. Bonnet, M. A. Siegler, J. Sánchez Costa, G. Molnár, A. Bousseksou, A. L. Spek, P. Gamez, and J. Reedijk, Chem. Commun. 5619 (2008).

${ }^{34}$ R. Boehler and K. De Hantsetters, High Press. Res. 24, 391 (2004).

${ }^{35}$ G. J. Piermarini, S. Block, J. D. Barnett, and R. A. Foremann, J. Appl. Phys. 46, 2774 (1975).

${ }^{36}$ CrysAlisPro, Oxford Diffraction Ltd., Version 1.171.33.66.

${ }^{37}$ G. M. Sheldrick, Acta Crystallogr. A64, 112 (2008).
${ }^{38}$ O. V. Dolomanov, L. J. Bourhis, R. J. Gildea, J. A. K. Howard, and H. Puschmann, J. Appl. Crystallogr. 42, 339 (2009).

${ }^{39}$ R. J. Angel, J. Appl. Crystallogr. 37, 486, (2004).

${ }^{40}$ A. Katrusiak, Acta Crystallogr. A64, 135 (2008).

${ }^{41}$ G. Molnár, T. Guillon, N. Ould Moussa, L. Rechignat, T. Kitazawa, M. Nardone, and A. Bousseksou, Chem. Phys. Lett. 423, 152 (2006).

${ }^{42}$ A. Eiling and J. S. Schilling, J. Phys. F 11, 623 (1981).

${ }^{43}$ A. Bousseksou, G. Molnár, and G. Matouzenko, Eur. J. Inorg. Chem. 4353 (2004).

${ }^{44}$ V. Ksenofontov, H. Spiering, A. Schreiner, G. Levchenko, H. A. Goodwin and P. Gütlich, J. Phys. Chem. Solids 60, 393 (1999).

${ }^{45}$ E. König, G. Ritter, J. Waigel, and H. A. Goodwin, J. Chem. Phys. 83, 3055 (1985).

${ }^{46}$ V. Ksenofontov, G. Levchenko, H. Spiering, P. Gütlich, J.-F. Létard, Y. Bouhedja, and O. Kahn, Chem. Phys. Lett. 294, 545 (1998).

${ }^{47}$ P. Gütlich, V. Ksenofontov, and A. B. Gaspar, Coord. Chem. Rev. 249, 1811 (2005).

${ }^{48}$ E. König, G. Ritter, W. Irler, and H. A. Goodwin, J. Am. Chem. Soc. 102, 4681 (1980).

${ }^{49}$ A. Bousseksou, J. Nasser, J. Linares, K. Boukheddaden, and F. Varret, J. Phys. I (France) 2, 1381 (1992).

${ }^{50}$ M. Nishino, K. Boukheddaden, S. Miyashita, and F. Varret, Phys. Rev. B 68, 224402 (2003).

${ }^{51}$ B. Hôo, K. Boukheddaden, and F. Varret, Eur. Phys. J. B 17, 449 (2000).

${ }^{52}$ K. Boukheddaden, J. Linares, R. Tamasa, and C. Chong, J. Phys.: Cond. Matter. 19, 106201 (2006).

${ }^{53}$ K. Boukheddaden, S. Miyashita, and M. Nishino, Phys. Rev. B 75, 094112 (2007).

${ }^{54}$ J. A. Nassa, K. Boukheddaden, and J. Linares, Eur. Phys. J. B 39, 219 (2004).

${ }^{55}$ W. Nicolazzi, S. Pillet, and C. Lecomte, Phys. Rev. B 78, 174401 (2008).

${ }^{56}$ H. Spiering, K. Boukheddaden, J. Linares, and F. Varret, Phys. Rev. B 70, 184106 (2004).

${ }^{57}$ T. Kohlhaas, H. Spiering, and P. Gütlich, Z. Phys. B 102, 455 (1997).

${ }^{58}$ H. Spiering, T. Kohlhaas, H. Romstedt, A. Hauser, C. BrunsYilmaz, J. Kusz, and P. Gütlich, Coord. Chem. Rev. 190-192, 629 (1999).

${ }^{59}$ D. Chernyshov, H.-B. Bürgi, M. Hostettler, and K. W. Törnroos, Phys. Rev. B 70, 094116 (2004).

${ }^{60}$ A. Das and B. Ghosh, J. Phys. C 16, 1799 (1983). 